\title{
Environnement et ruralité: enjeux et paradoxes
}

\section{Rural and the environment: word matters and paradoxes}

\author{
Jean-Paul BILLAUD*
}

\section{RESUMO}

\begin{abstract}
Os problemas ambientais são aqui analisados a partir da abordagem da ruralidade na perspectiva das ciências sociais. Três processos da ação política e coletiva são evocados: a construção de um espaço de sentido comum, a aplicação de conhecimentos diversos na normatização das atividades e a emergência de novos territórios do meio ambiente. O texto apresenta uma análise das sociedades rurais nos últimos trinta anos, evidenciando desafios e paradoxos marcantes no âmbito da modernidade.

Palavras-chave: ruralidades, meio ambiente, ciências sociais, território, patrimônio, modernidade.
\end{abstract}

\begin{abstract}
Environmental problems are analyzed here from a rural approach under the social sciences' perspective. Three political and collective action processes are addressed: the construction of a common sense space; the application of various kinds of knowledge in standardizing activities; and the emergence of new environmental territories. The text presents an analysis of rural societies in the past thirty years, highlighting striking challenges and paradoxes in the context of modernity.

Key-words: rural aspects, the environment, social sciences, territory, ownership, modernity.
\end{abstract}

Les problèmes que la société qualifie d'environnement sont pour certains inscrits au cœur de la ruralité où ils déplacent l'action politique (collective et publique) vers des objets multiples. Les sciences sociales s'intéressent plus particulièrement à la constitution des "collectifs" d'action qui, pour répondre aux problèmes posés, alimentent trois types de processus.

La construction d'un espace de sens commun tout d'abord, dans la mesure où les problèmes à traiter convoquent des intérêts divers, sinon contradictoires, des savoirs incertains ou refoulés, des valeurs dont le contenu doit être réexaminé ou inscrit sur une nouvelle échelle.

Le recours pour ce faire à l'expertise ou à une mobilisation de connaissances (scientifiques ou/et profanes) est constant, ce qui nécessite d'explorer la place de ces différents types de savoirs dans le guidage normatif des activités.

Et enfin, l'action collective, dans la mesure où elle reconfigure un espace public d'intervention à partir d'arènes plus ou moins structurées par des procédures, des débats

* Directeur de recherches - CNRS/LADYSS/PARIS 10, France. 
ou des controverses, est indissociable du territoire où elle se déroule. On est donc dans une situation où le territoire n'est pas prédéfini, car il est en quelque sorte coproduit par le traitement même du problème d'environnement.

C'est en ce sens que l'on peut parler de l'émergence, au cœur de ce que l'on désigne par ruralité, de nouveaux territoires de l'environnement : territoires où des identités sociales et collectives sont interrogées au même titre que les pratiques qui s'y déploient ; territoires où des modalités d'action sont à inventer pour intégrer des situations d'interdépendances écologique et sociale qui posent problème ; territoires où les frontières de l'action collective et publique sont donc redessinées, mais en devant toujours composer avec les cadres d'action institués.

Pour saisir ce qui se joue dans les sociétés rurales autour de la prise en charge des questions d'environnement, on retracera ce qu'on peut considérer comme des épisodes marquants, c'est-à-dire des moments où ce qui était stabilisé et durable n'apparaît plus comme tel. L'émergence des collectifs d'action, et leur analyse, est évidemment un indice fort de tels épisodes, mais l'expérience des acteurs ne se limite pas à de tels collectifs. Pour mieux saisir l'impact sur la ruralité de tels phénomènes, il est nécessaire de revenir rapidement sur les caractéristiques de cette dernière.

Le paradigme rural fondateur postule une correspondance entre un territoire, une production et un genre de vie (BILLAUD, 1999). Il a été mis en œuvre dans une double orientation qui, tout en associant la plupart du temps les mêmes chercheurs, n'a pas fait vraiment objet de convergence. La première orientation concerne la question paysanne. Il s'agit d'en évaluer la spécificité dans un contexte de modernisation. La seconde orientation porte sur les micro-sociétés rurales. Il s'agit là encore d'en analyser le statut dans un contexte d'intégration dans une société englobante.

Ces deux orientations ont des traits communs, caractéristiques de l'approche ruraliste: une référence constante à un cadre d'opposition entre ville et campagne; une question récurrente autour de la plus ou moins grande spécificité que l'on peut accorder aux propriétés des objets analysés; une assimilation non explicitée entre paysan et rural; une évaluation de l'entrée en modernité sous l'angle du changement technique et social; une actualité particulièrement vive des questions liée à la transformation des sociétés agraires de l'après-guerre, transformation qui s'avérera être un facteur de changement de la société toute entière.
L'agriculture et les agriculteurs comme groupe social vont ainsi concentrer sur eux - c'est tout du moins la représentation que l'on s'en fait - l'ensemble de ces traits. Ils deviennent le groupe de référence de la ruralité, antiurbain par excellence, mais perméable à l'innovation et acteur du changement. Autour des agriculteurs et avec eux, va se mettre en place un dispositif (FOUCAULT, 1997) - c'està-dire un ensemble hétérogène de pratiques, d'énoncés scientifiques, d'aménagements, d'institutions - qui a pour objectif l'entrée en modernité du paysannat et qui, dans le même temps, construit un nouveau type de ruralité. Le paysan et le rural ont alors la même attente à l'égard de la modernité, à savoir qu'elle soit équitable par rapport au monde urbain, mais qu'elle soit aussi respectueuse de leur spécificité.

Ce dispositif qui associe l'Etat, l'élite professionnelle, la recherche agronomique et technique ainsi qu'un appareil législatif et d'encadrement particulièrement imposant, est très robuste: il a une légitimité incontournable fondée sur sa capacité nourricière (de la France puis de la planète), tout en travaillant constamment à contenir les débordements qui l'affectent très rapidement: crises de surproduction (d'où l'instauration du système des quotas), crise de reproduction sociale (d'où la pluriactivité), crise budgétaire (négociation politique au niveau européen, mais sous veille constante du partenariat entre l'Etat et la profession agricole, ce que l'on nomme la cogestion), etc.

Dans ce contexte de l'après-guerre dominé par le traitement politique et social de la question agraire, la "crise environnementale" - entendons par là que certains débordements par rapport au dispositif sont qualifiés en tant que problèmes d'environnement - émerge en deux temps qu'il faut distinguer.

1) De 1970 à 1990, la question de l'environnement se constitue peu à peu en matrice des limites du projet agricole, mettant en critique sa capacité à contenir en lui la trajectoire des espaces ruraux. L'enjeu environnemental traverse alors l'ensemble de la société française (rappelons que le ministère de l'environnement est créé en 1971) et chacun des secteurs d'activité a sur ce plan une histoire spécifique. La ruralité est plus particulièrement marquée, en matière de protection de la nature, par la conception colbertienne de la protection forestière, qui, au nom de la raison d'Etat (en l'occurrence, le ministre de Louis XIV qui réserve la forêt domaniale à la production de bois d'œuvre 
pour la Marine nationale), chasse le paysan d'un espace essentiel à sa survie (CADORET, 1985). On s'en doute, la méfiance et même la défiance sont d'autant plus grandes que, d'emblée, le point de vue environnemental se présente comme un obstacle au projet modernisateur de l'agriculture et, par voie de conséquence, au renouveau de la ruralité.

Celle-ci, en particulier par le biais de l'aménagement du territoire, est façonnée par les impératifs du développement agricole, qui, dans le cadre de son dispositif orienté vers l'augmentation de la productivité de l'agriculture, aménage et réorganise les espaces ruraux. Electrification, alimentation en eau, remembrement, autant d'opérations concrètes qui prennent sens au sein du dispositif agricole, même si dans le même temps, on met à la disposition du développement de toute la société les conditions favorables de l'infrastructure, de l'urbanisation, de l'énergie et même du tourisme de masse.

L'approche fonctionnelle du territoire est la clé du réaménagement des espaces ruraux dans le cadre du nouveau dispositif agricole. Elle a plusieurs effets.

- Elle introduit tout d'abord une séparation radicale entre espaces productifs et espaces de protection. De ce point de vue, elle néglige les interdépendances entre les systèmes, travaille davantage à la clôture qu'à la perméabilité ou aux flux.

- Elle pose la question naturelle de façon spécifique. Soit celle-ci ne se pose pas, et alors on est totalement dans l'artefact et dans une perspective de "fin de la nature". Soit elle est renvoyée aux marges du système (d'où l'émergence des notions de "zone défavorisée" ou "campagne marginale" dans les années 70), ou bien circonscrite à des espaces spécialisés (les Parcs Nationaux dès les années 60 , soit le début de la modernisation agricole, ou dans la décennie suivante les réserves naturelles) à qui une relative autonomie de gestion est laissée.

- La création des Parcs Naturels Régionaux au début des années 70, puis l'émergence des notions (davantage liées à l'action qu'à la connaissance) comme la pluriactivité (sociale) ou le paysage (nature) sont autant d'amendements à un dispositif contraint, pour perdurer, à une réforme constante. Mais ils ne le subvertissent pas. L'agriculteur se fait un peu paysan, après avoir tenté de s'en démarquer totalement, l'unité de la profession où l'entrepreneur cohabite de plus en plus mal avec les petits agriculteurs apparaît de plus en plus factice, mais la reconfiguration des espaces ruraux reste subordonnée aux impératifs du dispositif agricole de la modernisation.

L'émergence de la question environnementale s'inscrit dans cette configuration selon les quatre modalités suivantes.

- Elle est, appliquée à l'agriculture, un sujet tabou dans l'espace public, s'il s'agit non pas de mettre en avant les aménités de la nature mais les dommages à la nature. Il faut que ce soit un marginal de la politique (Brice Lalonde, alors ministre de l'environnement) pour que ce tabou soit levé. Sa dénonciation publique de la pollution agricole est l'un de ces épisodes marquants qui permettent de repérer un tournant décisif dans la remise en cause du dispositif.

- Elle se matérialise le plus souvent dans des conflits d'appropriation (enjeux d'usages entre pratiques productives et pratiques de nature) entretenus par l'approche fonctionnelle de la gestion des territoires.

- Elle est perçue comme imprégnée des valeurs urbaines, plus particulièrement celles des couches moyennes qui de surcroît constituent la base militante de l'écologie politique, et les conflits qu'elle génère sont réinterprétés dans le paradigme traditionnel de l'opposition ville/campagne.

- Mais elle constitue un révélateur des ambiguïtés et même des paradoxes du dispositif au travers de deux phénomènes convergents: les porte-parole de la question environnementale n'ont de cesse de se réclamer de la spécificité du monde paysan et rural qui motive leur désir d'investissement naturaliste (c'est la trajectoire des néo-ruraux); les porte-parole de la critique de la modernisation agricole veulent réhabiliter la spécificité agricole et rurale et rencontrent sur ce terrain idéologique les associations environnementalistes (ces agriculteurs contestataires se nomment "paysanstravailleurs").

Cette première phase a deux effets sur le rapport entre environnement et ruralité: 
- Elle offre une image plurielle du rural, dans la mesure où la remise en cause du modèle agricole, même si son expression politique est limitée, est d'abord portée par ceux qui côtoient l'activité: les résidents secondaires souvent, mais aussi les ruraux confrontés à la dégradation de leur paysage, aux problèmes d'eau potable, etc. Il apparaît que la ruralité ne peut se réduire à sa seule composante agricole, elle-même divisée.

- Mais elle construit également une image plurielle de l'environnement, c'est-à-dire que celui-ci se révèle constitué de divers environnements : des aménités comme le paysage, des ressources naturelles qu'il faut entretenir et gérer, de la nature dont la conservation suppose une approche plus complexe que la simple mise en réserve, des risques majeurs qui rappellent la vulnérabilité des systèmes jusque dans l'habitat rural (inondation de zones construites par exemple, feux de forêt, etc.), puis peu à peu l'environnement global autour des questions du changement climatique. Ces environnements ne mobilisent pas toujours les mêmes acteurs, ils ne sont pas sujets au même degré d'incertitude, en un mot ils n'ont pas la même capacité à participer à la crise de la modernité. Ils sont cependant déterminants dans la mise en critique du projet moderne appliqué au secteur agricole et de sa prétention à être le porteparole de la ruralité.

2) A partir de 1990, la question naturelle s'impose, à savoir qu'elle acquiert une légitimité dans l'espace public, ce que traduit son intégration croissante dans la politique agricole européenne elle-même, désignée alors par politique agrienvironnementale (BILlaud, 2002). A cela, plusieurs raisons.

Il y a tout d'abord la crise agricole elle-même, en particulier le coût budgétaire exponentiel de la PAC, passé de 11,6 milliards d'euros en 1980 à 40 milliards aujourd'hui. La logique économique rejoint en cela la logique naturaliste dans la remise en cause, au nom du coût financier dans un cas et du coût écologique de l'autre, du modèle mis en place après la seconde guerre mondiale.

La crise environnementale elle-même, en prenant une toute autre ampleur, met en lumière les limites du dispositif agricole pour répondre aux grands enjeux du moment. On assiste en effet, à partir de la conférence de Rio en 1992, à une extension de la crise environnementale, en terme de changement d'échelle, dans l'espace (la planète) comme dans le temps (le développement durable). Autrement dit, une échelle spatio-temporelle qui montre que le dispositif de la modernisation agricole ne peut contenir toutes les interdépendances que de tels événements mettent en lumière.

Et enfin, plus fondamentalement, la crise environnementale apparaît de moins en moins comme une simple crise de croissance des sociétés à haute technologie, elle porte la marque d'une évolution cognitive et normative de grande ampleur. Cette évolution cognitive touche particulièrement l'agriculture et pose clairement la question du rapport entre l'agriculture et les écosystèmes. Pourquoi?

\section{De quelques enjeux...}

On peut aisément analyser le développement agricole et rural en tant que modalité de mise en œuvre du projet moderne.

D'une part, la modernisation agricole passe par un processus scientifique et technique d'affranchissement de l'activité productive à l'égard des contraintes du milieu physique. Apports d'engrais et de pesticides, façonnage de l'espace au profit de la mécanisation (agrandissement des parcelles, suppression des haies et des fossés, etc.), mise en place des stabulations libres en élevage, autant d'innovations dont l'objectif est la création d'une natureartefact qui va de la culture hors-sol jusqu'à l'animal mécanique.

De l'autre, le traitement de la nature s'inscrit tout autant dans le projet moderne. La séparation radicale dans l'aménagement des territoires entre espace de production et espace de protection participe de l'instauration du grand partage entre société et nature, si typique de la modernité occidentale. Les Parcs Nationaux et les réserves deviennent des champs d'application de l'écologie odumienne, basée sur les notions d'équilibre et d'isolat des systèmes. Les Parcs Naturels Régionaux, créés dans les années 70, seront des lieux assez conflictuels en raison de leur volonté de conciliation entre le développement et la conservation. Ils seront dans le même temps un lieu d'innovation favorisant la mise en critique des présupposés guidant la relation entre l'agriculture et la nature.

On peut également aisément analyser l'émergence des problèmes d'environnement dans les espaces ruraux comme 
l'expression de la question naturelle et comme une entrée en crise du projet moderne. Les transformations techniques qui sont au cœur de ce projet, à savoir l'innovation, exercent des effets en retour, inattendus, qui relèvent de processus naturels produits par ces mêmes transformations. C'est le prion qui s'invite chez la vache à 10.000 litres de lait annuels, les métaux lourds dans le sol qui interpellent la chimie, le nitrate qui devient déchet, le risque sanitaire qui s'introduit dans la chaîne de l'alimentation humaine. L'effort remarquable de l'artificialisation correspond aussi à une réintroduction du processus naturel.

Et enfin, les "objets de nature" que la crise environnementale fait émerger dans les espaces ruraux ont la particularité d'appartenir à ce que l'on nomme les "biens communs" l'eau avec ses enjeux de rareté, de qualité et d'appropriation; les ressources naturelles dans leur dimension renouvelable (la pêche, le bois) ou dans leur caractère d'irréversibilité (le et ses problèmes d'érosion, de contamination, de salinisation, de désertification, etc.).

Autrement dit, la question naturelle ne peut-être réduite à des enjeux d'appropriation, de distribution des activités sur un territoire. Elle met en scène des problèmes beaucoup plus complexes (la gestion des sols pollués; le suivi et l'évaluation des pesticides), objets d'incertitude sur le plan scientifique (les Organismes Génétiques Modifiés pour citer un cas de controverse) et l'agriculture apparaît comme un secteur d'activité qui est au cœur des enjeux parmi les plus cruciaux du moment, ceux qui, avec l'impact des biotechnologies, le génie génétique etc., relèvent de la question du vivant.

Il n'est plus pensable, dans un tel contexte, que l'agriculture puisse régler ses problèmes dans la famille de la cogestion, base de son dispositif, lorsque son activité la plus privée, la plus localisée peut mettre en péril la santé des populations (la pollution de l'eau potable) ou les équilibres planétaires (le méthane des rizières ou de l'élevage extensif et l'effet de serre). C'est pourquoi la crise du dispositif agricole affecte en premier lieu les mécanismes de la représentation de la profession et sa place dans la définition des politiques agricoles et rurales. Il s'agit avant tout que la diversité sociologique de la ruralité, allant du chasseur à l'écologiste, de l'agriculteur au résident ou au touriste, soit partie prenante (stakeholder en anglais) dans les enjeux auxquels doivent faire face les sociétés façonnées par le projet moderne.

Ainsi, ce qui se joue actuellement dans les nombreuses scènes de concertation autour du traitement de l'agriculture ou/et de la nature, c'est la capacité des acteurs ruraux à faire de l'environnement une "figure centrale du bien commun" (BOLTANSKI-THÉVENOT, 1991). Cela passe par un véritable travail politique, c'est-à-dire tout ce qui attise le sens critique, éthique ou le sens moral des acteurs ruraux et qui vaut discussion. Il y a là tout un secteur d'innovation sociale autour des apprentissages collectifs qui relève d'une autoproduction de la société. Mais le processus est complexe, difficile à appréhender car rarement univoque et encore exceptionnel. Son analyse passe d'abord par des études de cas (c'est-à-dire là où se fait ce travail politique) dont il faudra dire en quoi elles témoignent d'un changement profond et radical de la ruralité, confrontée à la résolution des questions d'environnement.

\section{A quelques paradoxes...}

D'ores et déjà, l'observateur ne peut que relever certains paradoxes.

- La dynamique de l'agriculture est tout à la fois celle d'une reterritorialisation (l'acte technique tend à prendre de plus en plus en compte, grâce à de nombreuses innovations, les conditions écosystémiques locales) et d'une globalisation dont le processus est alimenté tout autant par le marché que par l'environnement, ambivalence sinon ambiguïté que l'on retrouve dans la notion d'agriculture durable. Celle-ci ne renvoie-t-elle pas à un modèle d'innovation technique et de régulation des marchés par l'imposition de prix mondiaux (dont l'écoconditionnalité) pour les uns; à un modèle patrimonial basé sur la concertation et les aides directes pour les autres.

- La multiplication des procédures de concertation (l'enjeu est de construire des cahiers des charges pour les propriétaires ou les exploitants agricoles permettant des ajustements au plus près entre la production agricole et la conservation de la nature) ne doit pas faire oublier l'extension des normes (des règlements encadrant tous les aspects de l'activité à la requalification des espaces et des activités à partir de la référence écologique (cf. les politiques de traitement de la biodiversité). Cette reconfiguration des activités a pour objectif la production d'espaces écologiques (jusqu'à la restauration des zones humides par exemple) mais 
aussi une soumission croissante à la logique du marché mondial (cf. la Directive Cadre-Eau européenne).

Au-delà de ces paradoxes que l'on pourrait multiplier, on retrouve une constante liée à une double caractéristique. Il s'agit toujours de retisser le lien avec le territoire (le succès des notions de terroir ou la requalification naturaliste des espaces y contribuent par exemple), mais également d'instituer de nouveaux champs de relation et d'organisation qui débordent des cadres traditionnels de l'action collective en milieu rural. On voit ainsi apparaître de nouveaux territoires de gestion qui sont en décalage avec les modes de gouvernabilité habituels qui organisent les espaces ruraux; ils peuvent s'en démarquer ou s'y opposer (c'est souvent le cas pour la gestion de l'eau), ou bien les conforter en participant de leur renouveau (c'est par exemple le cas avec la notion de "pays" qui donne son sens au regroupement de communes traditionnelles).

Tout ceci renvoie à un certain type de modernité proposé à la ruralité.

Après avoir été organisée autour de l'impératif de la productivité agricole, elle doit intégrer les nouveaux enjeux de la maîtrise du vivant, à savoir une nouvelle complexité tributaire d'une innovation technologique fondée davantage sur la biologie que sur l'agronomie. A l'agriculteur-entrepreneur représentant de l'excellence professionnelle du dispositif agricole de l'après-guerre, devrait se substituer l'agriculteur high-tec, pour lequel l'information est un intrant plus déterminant que celui proposé par la chimie.

La remise en cause des modes traditionnels de gouvernabilité va de pair avec la montée en puissance de "dispositifs socio techniques" dans lesquels l'expertise, la mobilisation des connaissances sont déterminantes (MORMONT, 1996). D'où une généralisation de l'utilisation des SIG dans l'action publique, l'émergence de nouveaux acteurs de la médiation (environnementale, mais pas seulement), des asymétries d'information ou de pouvoirs liées à de nouvelles compétences, une extension de l'ingénierie écologique et de ses institutions.

Les forums de discussion, les systèmes de contractualisation sont autant de modes d'organisation de la vie en société qui renvoient à une certaine conception de la modernité, c'est-à-dire une démocratie procédurale (LASCOUMES, 1994) fondée sur le respect de règles du jeu, et une production de normes à partir d'arrangements locaux engageant, par le contrat, les individus.
Ces dispositifs socio-techniques liés au traitement des problèmes d'environnement définissent ainsi des scènes locales qui reflètent la diversité sociologique des espaces ruraux, mais également qui introduisent un décalage entre ces espaces sociaux construits dans la logique instrumentale de traitement d'un problème (à partir d'un bassin versant par exemple) et les espaces traditionnels de gestion et de sociabilité, davantage orientés vers la construction des identités.

La crise du dispositif agricole qui a accompli le projet moderne est manifeste. L'affirmation de la problématique environnementale donne tout à la fois un contenu critique et une issue pour un nouveau dispositif. La nouvelle modernité qui se dessine, au travers des lois, des conflits, des controverses, des instruments, des procédures, des accords, etc. est soumise à la tension entre deux pôles. D'un côté, une nouvelle phase de modernisation, sorte d'amendement et de correction par rapport aux limites de la première, que l'on qualifiera de "modernité écologique" (SPAARAGEN). De l'autre, une rupture plus profonde, supposant un véritable dépassement cognitif et normatif, que l'on qualifiera de "modernité réflexive", dans la mesure où elle passe par une grande capacité d'autoproduction de la société (GIDDENS, 1987). La première est un pari sur l'innovation scientifique et technique, la seconde sur l'innovation sociale et démocratique. Selon l'un ou l'autre scénario, la ruralité ne sera pas la même.

Ces évolutions dessinent un nouveau programme de recherche pour les sciences sociales. Il va de soi qu'elles invitent également au croisement des regards disciplinaires, plus particulièrement entre les sciences humaines et les sciences de la vie, mais nous laisserons ce point, essentiel, de côté.

Ce programme suppose de s'engager sur des pistes classiques. Face à l'émergence de tels enjeux, les typologies des valeurs et des attitudes à l'égard de l'environnement ont leur place. Il s'agit d'identifier ce que recouvre une diversité potentielle, sachant que, par hypothèse, on peut s'attendre au brouillage des appartenances sociales et professionnelles dans un contexte qui pousse à la redéfinition des identités. Le processus de prise en compte des problèmes environnementaux fait apparaître de nouveaux acteurs et de nouvelles procédures dont il faut analyser la place dans les configurations anciennes. Participation, négociation, conflit, autant d'objets de recherche pour les sciences sociales, mais il est vrai que la construction des argumentaires, dans un contexte 
d'incertitude scientifique et d'indétermination sociale, mérite une attention particulière. Ici, on fera plutôt appel à la sociologie de la justification, et là, dans le cas de la construction des problèmes d'environnement, à la sociologie des sciences qui invite à l'analyse des réseaux socio-techniques (LATOUR, 1989).

Ce programme suppose également d'ouvrir des chantiers plus spécifiques.

Il s'agit tout d'abord de repenser les rapports entre le local et le global. Si la globalisation peut être perçue comme une pensée de l'interdépendance généralisée, elle est en cela indissociable d'un retour sur le local. Le recours au sein des sciences sociales à la notion de territoire, réservée plutôt à la géographie, répond à l'exigence d'un lien entre local et global. Les politistes parlent ainsi de "territorialisation des politiques publiques" pour se démarquer des approches "top down", de même que les sociologues, et en leur sein les ruralistes avant tout, réévaluent le rôle du territoire comme cadre de l'action collective, comme vecteur de construction des identités sociales, comme lieu de nouveaux apprentissages.

Il s'agit également d'approfondir la question de la durabilité, si prégnante dans les problématiques sociales et politiques de l'environnement. Là aussi, le recours à la notion de patrimoine rend compte de l'interrogation sur le rapport au temps dans la crise du projet moderne (MICOUD, 2004). Le patrimoine se présente ainsi comme une nouvelle catégorie, ou une catégorie revisitée, dont les sociétés usent pour qualifier les objets et les activités par rapport à une reconstruction du bien commun.

Le nouveau dispositif agricole tente de se redéfinir autour de la notion de multifonctionnalité. Il est en effet confronté à l'irruption de nouveaux objets de gouvernement (la pollution, la biodiversité, le risque, le vivant), à la nécessité de nouvelles alliances (les scènes de concertation sont l'un des lieux où elles se construisent), la multifonctionnalité représentant dans ce contexte un concept suffisamment ouvert pour redéfinir les activités et les identités, en particulier sur le plan professionnel.

L'activité agricole représente un modèle d'association entre des pratiques et des savoirs. Elle est un véritable laboratoire pour l'analyse des rapports entre science et société dans les situations de controverses, mêlant incertitude et expertise. On peut voir dans ces multiples scènes où le rapport à la nature est rendu discutable, l'apprentissage d'une démocratie technique (CALLON, 1998). Si tel est le cas, il faudra analyser ses liens avec la démocratie politique et représentative qui constitue la base de la ruralité. L'environnement entraîne chez celle-ci un déplacement de l'action politique et citoyenne vers des objets multiples (pollution, déchets, bruit, eau, etc.) qui sont l'objet d'un travail politique qui envahit l'espace du quotidien.

$\mathrm{Si}$, pour conclure, on se place du point de vue d'une refondation de l'approche ruraliste, rendue nécessaire par la crise des catégories conceptuelles mobilisées dans l'analyse du projet moderne, on retiendra l'émergence des deux notions dont nous avons parlé, à savoir le territoire et le patrimoine. S'il est vrai qu'elles ne sont pas circonscrites aux relations entre environnement et ruralité, elles y sont mises à l'épreuve, en raison de leur place dans l'histoire des sociétés rurales, de façon particulière et privilégiée.

Est-ce l'amorce d'un nouveau paradigme de la ruralité, dans lequel il faudrait tenir compte des multiappartenances des individus (en lieu et place de l'idée de population) et de leur confrontation à des situations de vulnérabilité qui mettent en jeu leur responsabilité? Quelle que soit la réponse, il est évident que la question de l'environnement interpelle nos catégories de pensée et d'action dont précisément la ruralité fait partie. C'est donc un chantier à la fois théorique et épistémologique qu'il faut ouvrir, mais, fidèle en cela à la tradition des études rurales, en partant des situations empiriques que génère le rapport en construction entre la ruralité et l'environnement. 
BILLAUD, J. P. Environnement et ruralite: enjeux et paradoxes

\section{RÉFÉRENCES CITÉES}

BILLAUD, J. P. Sociologie Rurale. In: ACOUN, A.; ANSART, P. Dictionnaire de Sociologie. Paris: Le Robert Seuil, 1999. p. 462-465.

. (Dir.). Environnement et gestion des territoires, Paris: La Documentation Française.

CALLON, M. Des différentes formes de démocratie technique. Annales des mines, p. 63-73, jan. 2002.

FOUCAULT, M. Histoire de la folie à l'âge classique. Paris: Gallimard. 1997.

GIDDENS, A. La constitution de la société. Paris: PUF, 1987.
LASCOUMES, P. L'éco-pouvoir, environnement et politique, Paris: La Découverte, 1994.

LATOUR, B. La science en action. Paris: La Découverte, 1989.

MICOUD, A. Des patrimoines aux territoires durables. Ethnologie et écologie dans les campagnes françaises, Ethnologie Française, p. 13-22, jan./mars 2004.

MORMONT, M. Agriculture et environnement: pour une sociologie des dispositifs, Economie Rurale, n. 236, p. 28-36, 1996. 